\title{
Safety and Short-term Efficacy of Licartin Combined with Conventional Transcatheter Hepatic Arterial Chemoembolization for Unresectable Hepatocellular Carcinoma
}

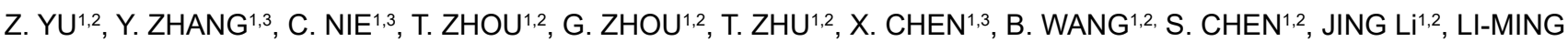 \\ WU $^{1,2^{*}}$, S. ZHENG ${ }^{1}$ AND J. SUN ${ }^{1,2,3^{*}}$ \\ ${ }^{1}$ Hepatobiliary and Pancreatic Interventional Treatment Center, The First Affiliated Hospital, Zhejiang University School of \\ Medicine, Hangzhou, Zhejiang Province, China, ${ }^{2}$ Zhejiang Clinical Research Center of Hepatobiliary and Pancreatic Disease \\ Hangzhou, Zhejiang Province, China, ${ }^{3}$ Zhejiang Provincial Research Center for Diagnosis and Treatment of Heapatobiliary \\ Diseases, Hangzhou, Zhejiang Province, China
}

Yu et al.: Safety and Short-Term Efficacy of Licartin

To evaluate the safety and short-term efficacy of Licartin combined with conventional transcatheterheaptic arterial chemoembolization for patients with unresectable hepatocellular carcinoma, 13 patients with unresectable primary liver cancer treated with Licartin combined with conventional transcatheter arterial chemoembolization at the First Affiliated Hospital from January 2012 to December 2014, were 
retrospectively analyzed. Licartin was infused via hepatic artery at a dose of $27.75 \mathrm{MBq} / \mathrm{kg}$ prior to conventional transcatheter arterial chemoembolization (pirarubicin $20 \mathrm{mg}$ and iodized oil), supplemented with poly vinyl alcohol particles. Here, the iodized oil is used as an embolic agent and carrier of anticancer drugs. After entering into the small arteries and peritumoral sinusoid of hepatocellular carcinoma through a catheter, the iodized oil gets retained there to block the terminal blood flow. According to mRECIST evaluation criteria, the remission and control rates after 1, 3, 6 , and 9 mo were 69.3 and $100 ; 69.3$ and $84.6 ; 61.5$ and 76.9 and $60 \%$ and $80 \%$, respectively. The survival rate was $100 \%$ after 6 mo. The 1 y and 2 y survival rates estimated with Life Tables were 68 and $45 \%$, respectively. Liver and kidney function showed no significant difference pre- and posttreatment. No serious infections, jaundice, gastrointestinal bleeding or bone marrow suppression occurred. Licartin combined with conventional transcatheter arterial chemoembolization showed short-term efficacy and minimal side-effects in patients afflicted with unresectable primary liver cancer. Long-term follow-up involving large cohorts is needed to determine the long-term efficacy of the intervention.

Key words: Licartin, transcatheter hepatic arterial chemoembolization, primary liver cancer, 131I-labeled metuximab

Hepatocellular carcinoma (HCC) is one of the most common primary lethal neoplasms worldwide ${ }^{[1]}$. Despite significant improvement in clinical diagnosis and management of HCC in the last decades, this malignant disease is still associated with poor prognosis and high postoperative recurrence rate ${ }^{[2,3]}$. Further, most HCC patients are contraindicated for radical treatment with surgical resection. Currently, transcatheter hepatic arterial chemoembolization (TACE) is the mainstay of treatment for patients with unresectable HCC. However, postoperative local recurrence and metastasis occur frequently, warranting the need for a long-term curative intervention. Combination of conventional TACE (cTACE) with radiotherapy has been shown to improve the therapeutic outcome. A lethal dose of conventional external irradiation is rarely administered to treat liver cancer due to limitations of cytotoxicity ${ }^{[4]}$. Licartinor ${ }^{131}$ I-metuximab binds with HAb18G (CD147) antigens, which are distributed on the tumor cell surface, to enhance the lethal effect via release of radiation ${ }^{[5]}$. However, its clinical efficacy and safety have yet to be established. The purpose of this study is to evaluate the safety and short-term efficacy of Licartin combined with conventional TACE for patients with unresectable HCC.

The medical records of 13 patients (13 males with a mean age of $59.77 \pm 14.58$ in the age range of 31-79 y) with unresectable liver cancer treated with Licartin combined with cTACE from January 2012 to December 2014 at the First Affiliated Hospital, were retrospectively reviewed. Patients with liver function graded Child-Pugh score A included 3 with hypertension, 10 with hepatitis $\mathrm{B}, 11$ with multiple lesions and 2 with single lesion. Three patients had portal vein tumor thrombus ( 2 involving the left branch and 1 the right branch). Nine patients carried portal vein tumors with a maximum diameter less than $5 \mathrm{~cm}$ and 1 patient had tumor measuring greater than $10 \mathrm{~cm}$ in diameter. The details of 13 patients are presented in Table 1.

TABLE 1: BASELINE PATIENT DEMOGRAPHICS

\begin{tabular}{lcc}
\hline Parameters & Quantitative value & Percent (\%) \\
\hline Gender & 13 & 100 \\
Male & 0 & 0 \\
Female & $31-79$ & \\
Age & & \\
Virus markers & 10 & 76.9 \\
HBsAg positive & 3 & 23.1 \\
HBsAg negative & 13 & 100 \\
Child-Pugh grade A & & \\
Portal vein tumor thrombus & 3 & 23.1 \\
Yes & 10 & 76.9 \\
No & & \\
Maximum diameter (cm) & 9 & 69.2 \\
$\leq 5$ & 3 & 23.1 \\
$>5, \leq 10$ & 1 & 7.7 \\
$>10$ & & \\
AFP value (ng/ml) & 5 & 38.5 \\
$\leq 10$ & 8 & 61.5 \\
$>10$ & & \\
Lesion number & 2 & 15.4 \\
Single & 11.6 \\
Multiple & & \\
\hline
\end{tabular}


Inclusion criteria were made after evaluating all cases based on the diagnostic criteria recommended by the Management of hepatocellular carcinoma ${ }^{[6]}$. Patients were diagnosed with primary liver cancer by needle biopsy, enhanced CT, MRI, ultrasound, DSA and AFP. Patients with a life expectancy greater than 3 mo. All cases diagnosed with multiple lesions or unresectable single lesion. All cases with no other dysfunction and all cases undergoing TACE alone with less reliable results.

Exclusion criteria were, patients who received ablation, targeted drug or other therapies; patients switching to or combined with other therapies for tumor progression after multiple sessions of TACE; patients with extra hepatic metastasis; patients complicated with serious underlying diseases and patients allergic to Licartin or its components. Lugol's liquid was administered $3 \mathrm{~d}$ before treatment until day 7 after treatment ( $0.5 \mathrm{ml}$ each time, 3 times daily). Skin test was carried out initially. Following confirmation of a negative response to subcutaneous injection of metuximab, an appropriate dose of ${ }^{131}$ I-metuximab was administered into the correct hepatic artery. Based on patients' weight, the amount of ${ }^{131} \mathrm{I}$ target dose was calculated as $27.75 \mathrm{MBq} / \mathrm{kg}(0.75 \mathrm{mCi} / \mathrm{kg})$.

After conventional preparation, bilateral inguinal disinfection and local anesthesia, radiography of the celiac trunk, common hepatic artery, superior mesenteric artery or inferior mesenteric artery was performed to determine the tumor location, size, number and blood supply in the right femoral artery using Seldinger's technique. A $2.7 \mathrm{~F}$ microcatheter was carefully advanced into the tumor feeding artery and Licartin was injected over a period of 5 to $10 \mathrm{~min}$, followed by embolization with iodized oil (1-20 ml) suspended in pirarubicin $(20 \mathrm{mg})$. Tumor size, blood flow, and degree of thickness dictated the speed of control injection. The iodized oil emulsion flow was monitored. The decision to stop injection was based on the sedimentary conditions of the iodized oil emulsion in the tumor area. The presence of small hepatic portal vein branches around the tumor was another factor. Polyvinyl alcohol particles were used in combination with iodized oil to occlude the feeding artery according to individual situation. Treatments protecting liver and stomach as well as antineoplastic interventions were routinely administered to patients. Antinausea, antipyretic and antiinfective treatments were used as needed.

Four weeks after treatment, CT or MRI, liver and kidney function tests, $\alpha$-fetoprotein (AFP) value, and routine blood evaluation were conducted to assess residual liver tumor and normal liver function. Local curative effect was divided into complete response (CR), partial response (PR), stable disease (SD), and progressive disease (PD). Disease remission rate was defined by CR combined with PR. Disease control rate was determined by the total remission rate and stable disease.

Ongoing follow-up was conducted in person at the outpatient clinic and inside the hospital, as well as remotely via telephone after the initial follow-up. The starting point of survival analysis was regarded as the day of initial treatment and the endpoint included death or until October 2015. All statistical analyses were conducted with SPSS version 13.0. Measurement data were analyzed by two related Wilcoxon test samples with $p<0.05$. Survival analysis was estimated in the postoperative $1 \mathrm{y}$, and the $2 \mathrm{y}$ survival rate using the life table method.

All patients were treated with Licartin combined with cTACE successfully after treatment with cTACE alone. Twelve patients were treated twice and the remaining patient underwent therapy once. The study involved 39 treatments, at an average of 3 times per patient. The average hospital time was $4.5 \mathrm{~d}$. The preoperative and postoperative ( 1 mo later) tumor sizes were $4.95 \pm 3.95$ and $3.8 \pm 3.23 \mathrm{~cm}$, respectively, and the differences were statistically significant $(\mathrm{p}<0.05)$. According to mRECIST evaluation criteria, the disease remission and control rates after $1,3,6$, and 9 mo were 69.3 and $100 \% ; 69.3$ and $84.6 \% ; 61.5$ and $76.9 \%$ and 60 and $80 \%$, respectively (Table 2 ).

All the 13 patients were followed up completely. The survival rate was $100 \%$ after 6 mo. The 1 - and 2 y survival rates estimated by Life Tables were 68 and $45 \%$, respectively. Six patients died by the end of follow-up and 7 patients were alive. The comparison of postoperative and preoperative AFP values showed no statistical significance $(\mathrm{p}=0.055)$. Changes in liver and kidney function, blood parameters, except serum

\section{TABLE 2: mRECIST CRITERIA FOLLOW-UP} RESULTS

\begin{tabular}{lccccccc}
\hline $\begin{array}{l}\text { Follow- } \\
\text { up time } \\
\text { (month) }\end{array}$ & Number & \multicolumn{4}{c}{ Tumor status } & \multicolumn{2}{c}{\begin{tabular}{c} 
(CR+PR \%) \\
\cline { 2 - 7 } (CR+PR+SD\%)
\end{tabular}} \\
\hline 1 & CR & PR & SD & PD & (CR+S & & \\
3 & 13 & 3 & 6 & 4 & 0 & 69.3 & 100 \\
6 & 13 & 4 & 5 & 2 & 2 & 69.3 & 84.6 \\
9 & 13 & 4 & 4 & 2 & 3 & 61.5 & 76.9 \\
9 & 10 & 4 & 2 & 2 & 2 & 60.0 & 80.0 \\
\hline
\end{tabular}


creatinine, revealed no significant pre and postoperative (1 mo) differences (Table 3).

The clinical symptoms were carefully recorded after treatment (Table 4). Frequent adverse events included nausea, vomiting, abdominal pain, anorexia, and fever, which were improved after symptomatic treatment. No liver or kidney failure, severe infection, gastrointestinal bleeding or other serious complications was observed.

${ }^{131} \mathrm{I}$, with a half-life of $8 \mathrm{~d}$, emits $\gamma$ and $\beta$ rays. However, only $\beta$ radiation is associated with therapeutic effect. The energy of $\beta$ rays is $364 \mathrm{Kev}$ and the radiation range is less than $1 \mathrm{~mm}$, which is completely blocked by fat and muscle surrounding the liver. Therefore, ${ }^{131}$ I therapy for liver cancer is highly efficient with low toxicity ${ }^{[7]}$. However, delivery of ${ }^{131} \mathrm{I}$ and enrichment in the tumor microenvironment are always a challenge. Internal radiation therapy with ${ }^{131}$ I-labeled lipiodol for HCC is effective ${ }^{[8]}$. However, non-specific ${ }^{131} \mathrm{I}$ accumulation in the tumor is a limitation and lipiodol is easily excreted. Licartin is a new ${ }^{131}$ I-labeled monoclonal antibody recommended for targeted radiation therapy of HCC. ${ }^{131}$ I-metuximab has been shown to exhibit antitumor efficacy by delivering radioactive ${ }^{131} \mathrm{I}$ to the tumor location by specifically binding to HAb18G (CD147) antigen, which is expressed on the tumor cell surface, Antibody-dependent cellular cytotoxicity is another mechanism. ${ }^{131} \mathrm{I}$-metuximab is effective against liver cancer since CD147 is expressed on HCC cells up to 70 to $80 \%{ }^{[9]}$. Biodistribution studies showed that Licartin was absorbed by liver cancer tissue and the radioactivity increased constantly over time resulting in persistent high-dose radiation delivery to tumor,

\begin{tabular}{|c|c|c|c|}
\hline Test items & Preoperative & Postoperative & $p$ value \\
\hline $\operatorname{AFP}(\mathrm{ng} / \mathrm{ml})$ & $1125.52 \pm 2986.9$ & $604.47 \pm 1572.76$ & 0.055 \\
\hline TBIL (BIL57 \pm 1$)$ & $15.92 \pm 7.15$ & $16.92 \pm 6.60$ & 0.552 \\
\hline AST (U/l) & $58.92 \pm 38.39$ & $47.38 \pm 13.3$ & 0.388 \\
\hline ALT (U/L) & $60.69 \pm 60.97$ & $34.15 \pm 10.62$ & 0.382 \\
\hline ALB $(g / l)$ & $37.09 \pm 5.19$ & $38.90 \pm 5.34$ & 0.279 \\
\hline Cre $(\mu \mathrm{mol} / \mathrm{l})$ & $67.62 \pm 13.21$ & $62.38 \pm 13.41$ & 0.041 \\
\hline WBC $\left(10^{9} / \mathrm{l}\right)$ & $5.96 \pm 3.15$ & $4.46 \pm 1.95$ & 0.346 \\
\hline PLT $\left(10^{9} / \mathrm{l}\right)$ & $111.77 \pm 45.52$ & $123.00 \pm 60.00$ & 0.552 \\
\hline
\end{tabular}

TABLE 4: CLINICAL SYMPTOMS IMMEDIATELY AFTER TREATMENT

\begin{tabular}{lccccc}
\hline $\begin{array}{l}\text { Adverse Abdominal } \\
\text { event }\end{array}$ & pain & Fever & $\begin{array}{c}\text { Nausea } \\
\text { vomiting }\end{array}$ & $\begin{array}{c}\text { Fatigue } \\
\text { anorexia }\end{array}$ & $\begin{array}{c}\text { Diarrhoea } \\
\text { constipation }\end{array}$ \\
\hline Number & 12 & 6 & 8 & 10 & 3 \\
$\%$ & 92.3 & 46.2 & 61.5 & 76.9 & 23.1 \\
\hline
\end{tabular}

without damaging the surrounding tissue ${ }^{[5]}$. Licartin combined with conventional TACE may benefit patients with $\mathrm{HCC}$ by prolonging the concentration of ${ }^{131} \mathrm{I}$ in the $\mathrm{HCC}$ tissues. It acts synergistically to enhance the radio sensitivity of chemotherapeutic drugs.

Lu et al. ${ }^{[10]}$ reported that the objective response rates of the 2 treatments at 4 to $8 \mathrm{w}$ after therapy were 69.1 and $62.8 \%$, respectively. The response rate tended to be significantly higher in the combination group than in the conventional therapy group. Another study reported similar results suggesting that the effective rates of the two groups after 1 mo of, treatment were 71.23 and $38.89 \%$. The present study also found that Licartin combined with cTACE for patients with unresectable HCC was effective in controlling the tumor locally ${ }^{[1]}$. The disease remission and control rates after 1, 3, 6, and 9 mo were 69.3 and $100 \%$; 69.3 and $84.6 \%$; 61.5 and $76.9 \%$; and 60 and $80 \%$, respectively. The postoperative and preoperative AFP values showed no significant differences and relates to fewer number of cases.

In the current study of Licartin combined with cTACE for unresectable HCC and post-operative recurrence, the curative effect was local and resulted mostly in short-term survival. The results showed that combination therapy showed survival rates of $88.2 \%$, $79.1 \%$ and $57.4 \%$ at 6,12 , and $18 \mathrm{mo}$, respectively ${ }^{[10]}$. The survival rates of HCC patients treated with cTACE alone were $81.6 \%, 67.4 \%$ and $38.6 \%$, respectively, and the difference was statistically significant. In another study the combination group at 6,9 and 12 mo showed significant survival rates of $86.4,74.1$ and $60.5 \%$ compared to $60.0,45.2$ and $34.4 \%$ in the control group, respectively. In the present study, the postoperative survival rate was $100 \%$ at $6 \mathrm{mo}, 1 \mathrm{y}$ and $2 \mathrm{y}$, and the survival rates estimated by Life Tables were 68 and $45 \%$, respectively ${ }^{[1]}$. By the follow-up end point, a total of 6 patients died, and 7 patients survived. The above data showed that the combination treatment was superior to cTACE for unresectable HCC.

All patients complained of gastrointestinal symptoms after the treatment, including nausea, vomiting and stomach ache, low-level fever and diarrhoea were less frequent and managed with symptomatic treatment. No liver or kidney failure, severe infection, gastrointestinal bleeding or other serious complications were observed. Evaluation of liver and kidney function revealed no major changes in blood parameters, except for serum creatinine. The preoperative results showed no 
significant differences compared with postoperative results at 1 mo. The probability of bone marrow suppression due to ${ }^{131} \mathrm{I}$ irradiation therapy was higher compared with TACE alone. However, the incidence of bone marrow suppression was not observed in this study. The risk associated with bone marrow suppression was reported by other studies ${ }^{[10-12]}$. The incidence of bone marrow suppression was mainly level I or level II. Findings similar to this study were reported by other researchers $^{[13]}$. The discrepancy may be patient-specific or related to the drugs used. Current study demonstrated that Licartin was safe for patients with unresectable and recurrent HCC.

In conclusion, the findings of this study indicated that the combination of Licartin and cTACE is an effective treatment for patients diagnosed with unresectable HCC. The adverse side effects were minor. The efficacy was local and superior to the individual therapy. The principal limitation of this study relates to its small sample sizeas well the overall survival rate is limited to $68 \%$ and $45 \%$ respectively in the first and second year. On the other hand the study depicts, that the treating surgical oncologists has tried vigorously to improve the survival rate Therefore, long-term, prospective, controlled and randomized studies with large sample sizes are needed.

\section{Acknowledgements:}

The present work was funded by Zhejiang Provincial Natural Science Foundation of China (Grant No.LZ18H180001), National Natural Science Foundation of China (Grant No. 81971713), National S\&T Major Project of China (NO.2018ZX10301201), Grant from Health Commission of Zhejiang Province (JBZX-202004), Research Unit of Collaborative Diagnosis and Treatment For Hepatobiliary and Pancreatic Cancer, Chinese Academy of Medical Sciences(2019RU019), The Key Research Development Program of Zhejiang province (Grant No.2018C03018), Key Science and Technology Program of Zhejiang province(No.WKJ-ZJ-1923) and National Key R\&D Program of China (No.2017YFC0114102).

\section{Informed consent:}

Informed consent was obtained from all individual participants included in the study.

\section{Conflict of interest:}

The all authors report no conflicts of interest in this work.

\section{REFERENCES}

1. Torre LA, Bray F, Siegel RL, Ferlay J, Tieulent JL, Jemal A. Global cancer statistics, 2012. Cancer J Clin 2015;65:87-108.

2. Ueno K, Miyazono N, Inoue H, Nishida H, Kanetsuki I, Nakajo M. Transcatheterarterial chemoembolization therapy using iodized oil for patients with unresectable hepatocellular carcinoma: evaluation of three kinds of regimens and analysis of prognostic factors. Cancer 2000;88:1574-81.

3. Llovet JM, Bruix J. Systematic review of randomized trials for unresectable hepatocellular carcinoma: chemoembolization improves survival. Hepatol 2003;37:429-42.

4. Hawkins MA, Dawson LA. Radiation therapy for hepatocellular carcinoma: From palliation to cure. Int J Am Cancer Soc 2006;106:1653-63.

5. Zhang Z, Bian H, Feng Q, Mi L, Mo T, Kuang A, et al. Biodistribution and localization of iodine-131 labeled Metuximab in patients with hepatocellular carcinoma. Cancer Biol Ther 2006;5:318-22.

6. Bruix J, Sherman M. American Association for the Study of Liver Diseases. Management of hepatocellular carcinoma: an update. J Hepatol 2011;53:1020-2.

7. Chen $\mathrm{ZN}, \mathrm{Mi} \mathrm{L}, \mathrm{Xu}$ J. Targeting radioimmunotherapy of hepatocellular carcinoma with iodine $\left({ }^{131} \mathrm{I}\right)$ metuximab injection: clinical phase I/II trials. J Int J Radiat Oncol Biol Phys 2006;65:435-44.

8. Kobayashi H, Nakajo M, Shimabukuro K, Shirono K, Sakata $\mathrm{H}$, Oyama $\mathrm{T}$, et al. Possibility of radiotherapy of hepatic cancer by transcatheter arterial embolization with radioactive Lipiodol. Chin J Cancer Res 1984;44:96.

9. Mamori S, Nagatsuma K, Matsuura T, Ohkawa K, Hano H, Fukunaga M, et al. Useful detection of CD147 (EMMPRIN) for pathologicaldiagnosis of earlyhepatocellularcarcinoma in needlebiopsysamples. World J Gastroenterol 2007;13:2913.

10. Wu L, Yang YF, Ge NJ, Shen SQ, Liang J, Wang Y, et al. Hepaticartery injection of ${ }^{131}$ I-labelled metuximab combined with chemoembolization for intermediate hepatocellular carcinoma: a prospective nonrandomized study. Eur J Nucl Med Mol Imaging 2012;39:1306-15.

11. He Q, Lu WS, Liu Y, Guan YS, Kuang AR. ${ }^{131}$ I-labeled metuximab combined with chemoembolization for unresectable hepatocellular carcinoma. World J Gastroenterol 2013; 19:9104.

12. Wu L, Yang YF, Ge NJ, Shen SQ, Liang J, Wang Y, et al. Hepatic Arterial Iodine- ${ }^{131}$ I-Labeled Metuximab Injection Combined with Chemoembolization for Unresectable Hepatocellular Carcinoma: Interim Safety and Survival Data from 110 Patients. Cancer Biother Radiopharm 2010;25:65763.

13. Li Z, Zhou JX, Ren JZ, Zhang WJ, Han XW. Clinical value of iodine $\left[{ }^{131} \mathrm{I}\right]$ metuximab infusion combined with TACE for treatment of patients with post-intervention relapse of mid or advanced stage hepatocellular carcinoma. Chinese J Hepatol 2013;21:728-33.

This is an open access article distributed under the terms of the Creative Commons Attribution-NonCommercial-ShareAlike 3.0 License, which allows others to remix, tweak, and build upon the work non-commercially, as long as the author is credited and the new creations are licensed under the identical terms

This article was originally published in a special issue, "Biomedical research applications in Pharmaceutical Sciences" Indian J Pharm Sci 2020:82(2)spl issue2;40-44 